\title{
Optical Fiber Grating Sensor Data Access Technology for GPON
}

\author{
Han Qiang \\ China Satellite Maritime Tracking and Control \\ Department \\ Jiangyin, China \\ 1510603630@qq.com
}

\author{
Ni Hanjian \\ China Satellite Maritime Tracking and Control \\ Department \\ Jiangyin, China \\ 2794049921@qq.com
}

\begin{abstract}
In order to realize the remote transmission of sensing information in optical fiber sensing network, and ensure that the information data is not destroyed in the transmission process, the feasibility analysis of FBG (fiber grating sensor network) into GPON (passive optical network) is carried out. Comprehensive consideration, GPON can access a variety of services and adaptation mode, the sensor multiplexing frame end use of optical network unit of Fast Ethernet port access to GPON transmission. Using MATLAB simulation software of sensor network data the channel is transmitted to the remote monitoring center and the sensing data demodulation, compared the sending data and receiving data sequence of terminal, analyzes the bit error rate (BER) eye diagram parameters, verify the sensing information access GPON and has feasibility in the remote monitoring center demodulation.
\end{abstract}

Keywords-Optical fiber grating sensor; GPON; Data adapter; DBA; Access

\section{INTRODUCTION}

Fiber Bragg grating sensor is not affected by the power energy, it can be more reliable to collect the data of the monitoring environment, which is more reliable and safe. If the sensing information can be transmitted to the remote data processing, it can solve the problem that is not suitable for the on-site monitoring. Optical fiber sensing network, the fiber optic channel is not only difficult to construction, but also high cost. Therefore, the idea of the sensor network and the existing communication network is proposed, and the sensing signal is transmitted through the existing mature technology.

\section{FIBER BRAGG GRATING SENSOR}

Fiber Bragg grating can be seen as a kind of narrow band transmission filter or reflector. The change of refractive index is formed by the interaction of the incident photon and the germanium ion in the core. The incident light emitted by a broad spectrum light source will be returned, and the light of the other wavelength of the wavelength of the B will be transmitted. When the phase matching condition is satisfied, the reflection wavelength of fiber Bragg grating is determined by the coupling wave theory:

$$
\lambda_{B}=2 n_{\text {eff }} \Lambda
$$

$\lambda_{B}$-the Bragg wavelength of grating;

\author{
$2 n_{\text {eff }}$-effective refractive index of grating; \\ $\Lambda$-grating period.
}

On the two sides of the upper type of differential,

$$
\Delta \lambda_{E}=2 \Delta n_{\text {eff }} \Lambda+2 n_{\text {eff }} \Delta A
$$

The principle of fiber Bragg grating sensor: when the temperature, strain, pressure and other physical quantities are changed, the effective refractive index changes of the grating period or the core is changed, which causes the change of the central wavelength of the reflected light. The relationship between the central wavelength shift of fiber Bragg grating and temperature and strain is expressed as:

$$
\frac{\Delta \lambda_{E}}{\lambda_{B}}=\left(a_{f}+\varepsilon\right) \Delta T+\left(1-P_{E}\right) \Delta \varepsilon
$$

$a_{f}$-thermal expansion coefficient of optical fiber;

$$
\mathcal{E} \text {-thermal optical coefficient of material; }
$$

$\mathcal{E}$-the elastic optical coefficient of fiber material.

In all the Bragg wavelength shift caused by external factors, the most direct is the stress, strain and temperature parameter. Because of the tensile or compression of the fiber grating, it is bound to lead to the change of the grating period, and the optical fiber itself has a light effect, which makes the effective refractive index change with the change of the stress state. In the same way, the change of external temperature can also cause the change of the two parameters of the fiber grating period and effective refractive index. Therefore, the fiber Bragg grating is used to make the fiber optic sensor and temperature sensor.

\section{THE FEASIBILITY ANALYSIS OF SENSOR DATA TRANSMISSION IN OPTICAL ACCESS NETWORK}

\section{A. Light source}

Optical fiber communication system for the light source: the light wavelength and fiber low attenuation and reasonable dispersion wavelength is consistent with the high output power; spectral width is narrow, in order to reduce the transmission rate of the dispersion limit; easy and transmission fiber coupling; optical modulation is convenient; the work life is long, good stability, small size, light weight, low price, can be mass production, etc.. In optical fiber sensing network, the light source must have 
enough intensity and brightness; the volume of light source is small, and the optical fiber is coupled. The wavelength of optical fiber is low loss region. At present, the light emitting diode, semiconductor laser, amplified spontaneous emission light source, distributed feedback laser, quantum well semiconductor laser, $\mathrm{He} \mathrm{Ne}$ laser, etc.

\section{B. Transmission medium}

Optical fiber sensing network and optical fiber communication network are used as the transmission medium, the full use of the optical fiber transmission bandwidth, communication capacity, anti-electromagnetic interference ability, corrosion resistance, light weight and other advantages.

\section{Photoelectric detector}

In optical fiber communication applications, the photoelectric detector is required to have high photoelectric conversion efficiency, which is to be able to output as high as possible. The sensitivity is high, and the detection of weak signal is small, the signal distortion is small, the signal distortion is small, the reliability is high, and the size can be matched with the fiber diameter. In fiber grating sensing system, the detector is used for wavelength selectivity, so it is generally used in optical fiber sensing system, and its sensitivity, bandwidth and other parameters directly affect the overall performance of the fiber optic sensing system. In optical fiber sensor, the photoelectric detector is PIN photodiode, avalanche photodiode APD and CCD, etc.. Optical fiber sensing and optical fiber communication are similar to the light source, the transmission medium and the photoelectric detector. Also used in optical fiber communication devices, such as optical isolator, a coupler, multiplexers, and optical switch, etc. device can also be used in optical fiber sensing, so the realization of the two fusion is feasible.

\section{FIBER GRATING SENSOR SIGNAL ACCESS SYSTEM BASED ON GPON}

\section{A. GPON system structure}

Optical network terminal (OLT) and optical network unit (ONU) are connected by optical network (ODN). ONU can be used in a variety of ways to connect users, a ONU can connect multiple users. PON is a point to multipoint (P2MP) structure of passive optical network. GPON is a technology of PON, supports up to $1.25 \mathrm{Gbit} / \mathrm{s}$, the access rate of the downlink $2.5 \mathrm{Gbit} / \mathrm{s}$, supports the maximum physical distance $20 \mathrm{~km}$, the maximum logical distance $60 \mathrm{~km}$ over the long transmission distance. While supporting 1:64's split ratio, the expansion can support the 1:128's split ratio, with the advantages of a large number of users and a large coverage. Support: voice, data, video, leased line, distributed business. GPON supports high bandwidth transmission, which can effectively solve the bandwidth bottleneck of the twisted pair access, and meet the needs of high bandwidth services. GPON supports long distance access, which can solve the problem of long distance coverage of twisted pair access.

\section{B. Remote monitoring network topology of fiber grating sensing signal based on GPON}

GPON access optical fiber sensor signal, and then through the communication network to reach the remote monitoring center, monitoring center to receive the data frame analysis. In the user side, various types of sensors are used to measure stress, temperature, pressure and other environmental variables, the sensor will be collected in real-time transmission to the remote monitoring center, including sensor location information, sensor type and sensor data, etc.. A complete sensor network data frame is very limited, time resource is very rich, and for each sensor network to set up a separate interface access GPON is a waste of resources. So the sensor network data into the GPON before the access node processing. The access node uses fixed time division multiple access technology, and then is packaged into a fixed length time division multiplexing frame. The access node mainly completes the signal's code rate adjustment, fixed time division multiple access, the assembly and transmission of the sensing multiplexing frame, so that the sensing signal is suitable for transmission in the communication network, and the multiple sensing network is connected to the ONU port of GPON. In the GPON OLT through the shunt ratio splitter $1: \mathrm{N}$ and ONU connected. Each ONU is connected with a variety of user terminal equipment. In this paper, the optical fiber grating sensing service can be regarded as a fixed telephone or video service, and the access node processing system can be equivalent to a kind of user terminal equipment. The access node processing of sensor data, in time division multiplexing ONU port and other business again and through the same wavelength modulation, forming optical signal by the splitter for uplink transmission to the OLT. OLT receives the sensing data through the transmission network, and finally reaches the remote monitoring center. The remote monitoring center is responsible for sending all kinds of operation commands to control the behavior of the sensor site, collect the information, process the data frame and analyze whether the sensing data exceed the preset threshold value. Monitoring center has a strong information processing capability of the server, running the special development of monitoring and management software, can be the state of the monitoring network, and its storage, can be at any time to query the monitoring point of sensing information.

\section{GPON NETWORK ADAPTIVE SENSING SERVICE}

GPON defines a new transport sink layer (GTC), which consists of two parts: GTC framing sub layer and GTC adaptive sub layer. GPON system uses GTC protocol to achieve the integrated access to a variety of user services such as ATM, Ethernet, and TDM services. OAM is located at the top of the physical layer for the realization of physical layer of operation, management and maintenance functions, such as the realization of ONU registration and ranging, data encryption, ID allocation, port identifier allocation, error rate monitoring, state detection and other functions. The optical network terminal management and control interface (OMCI) is used to identify the virtual channel, virtual path and port identifier, and at the same time, the exchange between the OMCI channel data and the high level. GTC adapter layer 
includes 3 business adapters: OMCI adapter, ATM adapter, and GEM service adapter. In the GTC protocol stack, the OMCI information can be transmitted by the ATM service adapter or the GEM service adapter. GTC into the frame of the GEM frame and ATM cell assembly, forming GTC data frame, which has the functions of multiplexing, multiplexing, frame header creation and decoding, and internal routing based on port identifier.

Through GPON access network, the remote monitoring center sends sensor data to uplink data transmission, so it is suitable for GTC uplink burst data frame, the GPON protocol is used as a specific business, and then sent to the monitoring center by GEM adapter and GTC. GPON protocol as a sensor network as a single user, to achieve a transparent transmission of sensing data. Although the sensing data frame is fixed in a certain sense, the number of FBG sensor is enough for the actual sensing application, the data frame may be too large, and the data frame is segmented into multiple GEM frames. The PLI in the GEM frame determines the maximum length of the load to be 4095 bytes. If the length of the sensing data frame is greater than that value, it is necessary to segment the data. The GEM protocol has the function to segment the user data frame, and it can be divided into segments adjacent to any segment, and the end section of the GEM frame in the PTI frame indicates the end of the data frame.

\section{SIMULATION ANALYSIS OF FIBER OPTIC SENSOR NETWORK AND ACCESS NETWORK}

The sensor data is simulated and designed. Firstly, the optical signal is collected and converted into binary sequence by photoelectric conversion. Here we use the random binary sequence to represent the sensor information. Secondly, the sensing data packets of encoding are encapsulated in the sensing data packets, and then packaged into the sensing data frame. Finally, the sensing data frames are multiplexed to form a sensing multiplexing frame by using the time division multiplexing technology. Sensor receiving part of the sensory information to the sender with a demodulation, sensing information decoding removal sensor multiplexing frame of frame head and tail information, namely the sensor multiplexing frame encapsulation solution, the packaged sensor multiplexing frame digital tap, recovery code rate, frame, unpacking, decoding, and then draw the sensor specific information.

Eye (eye diagram) refers to using the experimental method, the estimation and improve the performance of the transmission system, a pattern observed on the oscilloscope. Through the eye to observe the effects of inter-symbol interference and noise, and then determine the pros and cons of the system, eye piece of the larger and more correct, said inter-symbol crosstalk is small, on the contrary it means the greater the inter-symbol crosstalk. When the system noise and the noise will be superimposed on the signal, resulting in blurred eye stitch, greater noise, line Jiyue wide increasingly blurred.

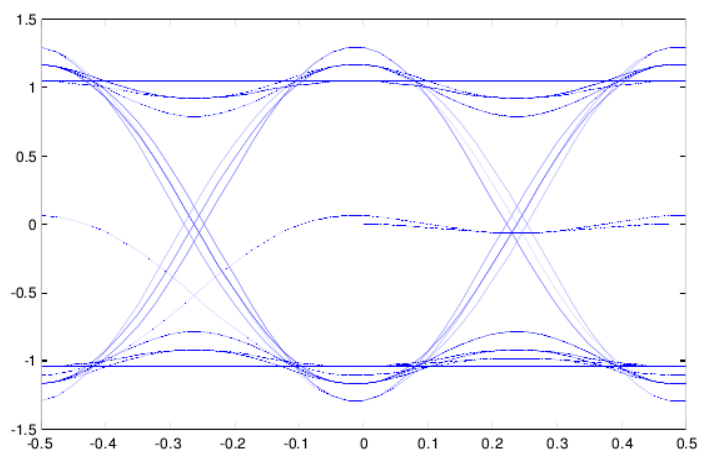

Figure 1. original signal eye diagram

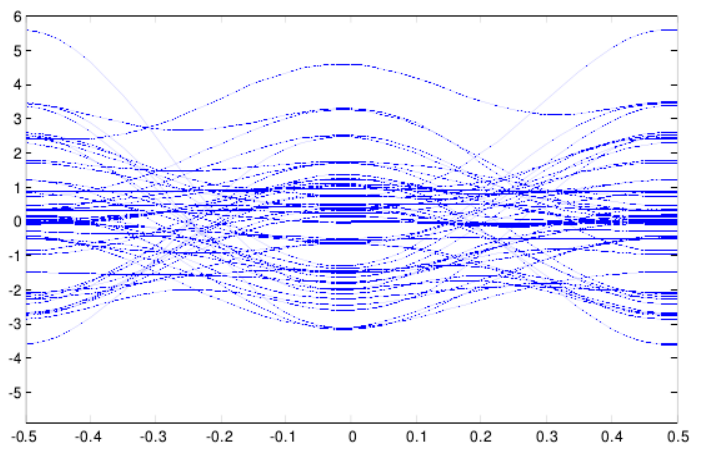

Figure 2. receiver with noise signal eye diagram 


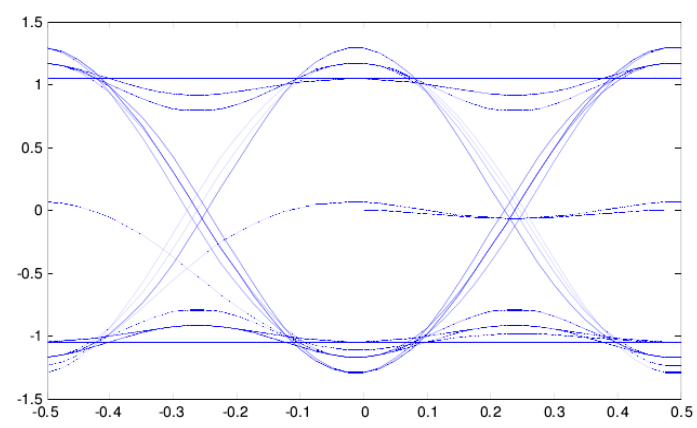

Figure 3. after decoding the signal eye diagram

Figure A is a primitive eye sensing subnet to send out a signal, the stitch is clear, and the eyes is very big, very good. Figure B is sensing monitoring center receives the sensing signal of the eye can be seen from the figure, when the signal reaches the monitoring center, due to the noise interference, the eye is destroyed. Figure $\mathrm{C}$ is a sensor monitoring center received after decoding, the signal eye diagram. From the above three eye effect comparison shows that, when sensing signal is received by the sensor monitoring and control center, through demodulation and decoding, can get the signal-to-noise ratio is relatively high, to enhance the overall performance of the system.

\section{CONCLUSIONS}

The feasibility of optical fiber sensing network and optical access network is analyzed from three aspects: the light source, the transmission medium and the photoelectric detector. The synchronization mechanism of GPON is analyzed, and a suitable scheme for GPON is proposed. Using MATLAB simulation tools, simulation modeling has been carried out on the optical fiber sensing net, eye and other parameters are obtained through the simulation, compared the sending end of the data and receive data terminal, and analyzed the performance of the system to verify the feasibility of fusion of optical fiber sensing network and communication networks.

\section{REFERENCES}

[1] Raymond, Bow thruster induced noise and vibration [C] Dynamic positioning committee, USA, 2000.

[2] Morey WW, Meltz G, Glenn W H. Fiber Optic Bragg Grating Sensors $[\mathrm{C}] / /$ Proceedings of Society of Photo-Optical Instrumentation Engineers, 1989, 1169: 98-107.
[3] Deming, Sun Qi true distributed optical fiber sensing technology and its application [J] Laser \& Optoelectronics Progress, 2009, 11: 29-33.

[4] bear Sen distributed fiber sensing technology into EPON research [D] Chengdu: University of Electronic Science and Technology, 2009.

[5] Ma Caihong integration of distributed optical fiber sensing technology and PON of [J] Electronic Science and Technology, 2011, 24 (9): 97-100.

[6] Hill KO, Y Fujii, Johnson DC, et al Photosensitivity in Optical Fiber Waveguides:. Application to Reflection Filter Fabrication [J] Applied Physics Letters, 1978, 32 (10):647-649.

[7] Murayama H, Igawa H, Omichi K, et al Application of Distributed Sensing with Longlength FBG to Structural Health Monitoring $[\mathrm{C}] / /$ Procceedings of 9 th International Conference onOptical Communications and Networks.China, nanjin, 2010:. 18-24

[8] Vendittozzi C, Sindoni G, Paris C, et al. Application of An FBG Sensors System for Structura Health Monitoring And High Performance Trimming on Racing Yacht [C]// Procceedings of 5th International Conference on Sensing Technology. New Zealand, $2011: 617-622$.

[9] Tube Super Fiber Grating Sensor Data Acquisition System and GPON frame structure for the [D] Zhucheng: Yanshan University Engineering Master's thesis, 2013,621.39

[10] Wang Yu-bao, Sea military fiber Bragg grating wave / sensor networks based on Time Division Multiplexing [J] Optics, 2010,30 (8): 2196-2201

[11] Li DS, Sui QM, Jia L. Simulation of Fiber Bragg Grating Sensing Networks Using Code Division Multiplexing and Wavelength Division Multiplexing [J] Acta Optica Sinica, 2010,30 (suppl): 100-105.

[12] Effenberger F, Cleary D, Haran O, et al An Introduction to PON Technologies [J] IEEE Communications Magazine, 2007,45 (3):S17-S25. 